\title{
Effects of the COVID-19 Pandemic on the Spiritual and Mental Well-Being of ICU Surrogates and their Treatment Preferences
}

\author{
Anthony Williams ${ }^{1}$, Alexia M Torke ${ }^{2,3,4}$, Emily S Burke ${ }^{2}$, James E Slaven ${ }^{5}$ \\ ${ }^{1}$ Indiana University (IU) School of Medicine; ${ }^{2} \mathrm{IU}$ Center for Aging Research, Regenstrief \\ Institute, Inc., Indianapolis, Indiana; ${ }^{3} \mathrm{U}$ Division of General Internal Medicine and Geriatrics, \\ Indianapolis, Indiana; ${ }^{4}$ Daniel F. Evans Center for Spiritual and Religious Values in Healthcare, \\ IU Health, Indianapolis, Indiana; ${ }^{5}$ IU Department of Biostatistics, IU School of Medicine, \\ Indianapolis, Indiana
}

Aspects of religiosity and spirituality (R/S) are associated with alleviating stress and influencing the treatment decision-making process in surrogates of patients in the intensive care unit (ICU). The COVID-19 pandemic has affected the mental health of the population, but no significance has been established with spiritual well-being. This study hypothesized that the pandemic negatively affected surrogate spiritual well-being in a relationship mediated by a surrogate's intrinsic religiosity, religious coping, and non-organizational religiosity. It was also hypothesized that surrogates would desire less aggressive treatments and have lower goals of patient function due to the pandemic. Baseline surrogate survey data using the FACIT-sp, Brief RCOPE and Durel scales, as well as answers to questions regarding treatment preferences and goal of patient function from the Chaplain Family Project were used in this study along with the pandemic status of the surrogate at the time of interview. There were 100 surrogates interviewed prior to the pandemic and 82 interviewed during the pandemic with a mean age of 54.5 and 53.8 years, respectively, who were mostly white $(82.0 \%$ and $75.6 \%$, respectively), female $(72.0 \%$ and $72.0 \%$, respectively), and on average had obtained at least an associate degree. There was a significantly greater preference for aggressive, life-sustaining interventions (LSIs) over relief of pain (26.1\% pre-pandemic to $40.9 \%$ during pandemic, $p=0.0494$ ) and a significantly fewer proportion of patient-surrogate advance conversations $(66.7 \%$ to $51.9 \%$, $p=0.0435$ ) in the during pandemic group. There was no statistical difference found in spiritual well-being, intrinsic religiosity, religious coping, non-organizational religiosity (NOR), treatment preferences, nor patient function goals between the pandemic status groups, when controlling for surrogate demographics and previous patient advance care planning. A longitudinal study should further investigate the possible effect of the pandemic upon surrogate spiritual well-being and ICU decisions and experiences for greater clarity. 